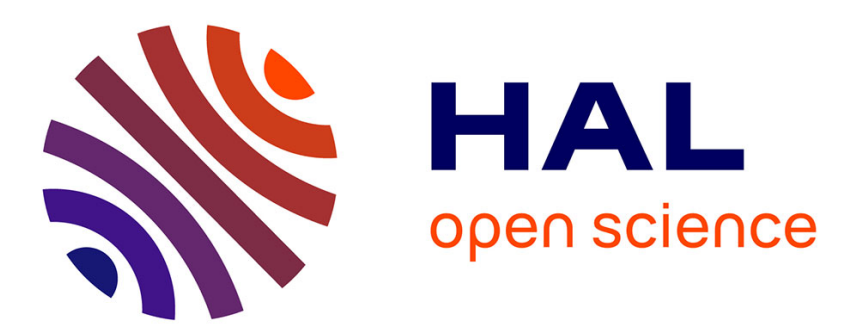

\title{
Multiple Time-Variant Targets Detection using MIMO Radar Framework for Cerebrovascular Monitoring
}

Mohammad Ojaroudi, Stephane Bila

\section{To cite this version:}

Mohammad Ojaroudi, Stephane Bila. Multiple Time-Variant Targets Detection using MIMO Radar Framework for Cerebrovascular Monitoring. 2021 15th European Conference on Antennas and Propagation (EuCAP), Mar 2021, Dusseldorf, Germany. pp.1-5, 10.23919/EuCAP51087.2021.9411329 . hal-03455450

\section{HAL Id: hal-03455450 \\ https://hal-unilim.archives-ouvertes.fr/hal-03455450}

Submitted on 29 Nov 2021

HAL is a multi-disciplinary open access archive for the deposit and dissemination of scientific research documents, whether they are published or not. The documents may come from teaching and research institutions in France or abroad, or from public or private research centers.
L'archive ouverte pluridisciplinaire HAL, est destinée au dépôt et à la diffusion de documents scientifiques de niveau recherche, publiés ou non, émanant des établissements d'enseignement et de recherche français ou étrangers, des laboratoires publics ou privés. 


\title{
Multiple Time-Variant Targets Detection using MIMO Radar Framework for Cerebrovascular Monitoring
}

\author{
Mohammad Ojaroudi ${ }^{1,2}$, Stéphane Bila ${ }^{1}$ \\ ${ }^{1}$ XLIM, UMR no7252, University of Limoges/CNRS, 123 Av. Albert Thomas, 87060 Limoges, France \\ ${ }^{2}$ Inria Lille Nord Europe, 40 Av. Halley, 59650 Villeneuve d'ascq, France \\ e-mail: mohammad.ojaroudi-parchin@inria.fr
}

\begin{abstract}
This paper presents a multi-input multi-output (MIMO) radar framework to detect the activated brain regions as a multiple target scenario for cerebrovascular monitoring applications. For this purpose, a setup of microwave brain monitoring system is designed to extract cerebrovascular information from the simulated full head phantom. The proposed setup includes multilayer brain phantom with varying blood vessel and bowtie antenna with precisely realized matching medium, which provides a fractional bandwidth from 0.5 to $5 \mathrm{GHz}$. In order to extract dynamic information from the blood vessel dilation, first, a single vessel with dilation properties, second two vessels located in different ranges and third two targets with different dilation rates are considered. The spectrogram and CFAR methods are used to show the vessel dilation and extract doppler information. Simulated results from all scenarios are presented to demonstrate the fruitfulness of the proposed method for precisely detecting the time-dependent characteristics of cerebrovascular targets.
\end{abstract}

Index Terms-multi-input multi-output (MIMO) radar, cerebrovascular monitoring, constant false alarm rate (CFAR), range-doppler profile, waveform diversity.

\section{INTRODUCTION}

The feasibility of using microwave-imaging system for diagnostics applications such as brain tumor and stroke detection, breast cancer detection, heart imaging, and bone imaging have been proved in various research reports [1-3]. There are two main categories in microwave imaging which are passive and active techniques. All of these techniques are in the near field imaging scenario, which is a very challenging area [4]. In this point of view, in microwave imaging system, reconstructing an image from estimated electrical filed distribution within a region of interest (ROI) is performed by using surface field observations on the of ROI, and this involves estimating the distribution of dielectric properties within the target volume. These techniques have the ability to reconstruct an image by mapping the relative energy (strong scattered) or dielectric coefficients of different points on the area to be imaged [5].

Microwave imaging system using multi-input multi output (MIMO) radars recently has been proposed as a non-invasive, low-cost and rapid modality for biomedical imaging applications [6-7]. The waveform diversity property of MIMO radars, can greatly enhance flexibiliry and performance capacity of the system [8]. There are many challenges in cerebrovascular monitoring using MIMO radars, such as detection of multiple-targets in the presence of clutter and high path-loss and waveform diversity and its advanteges in biomedical applications [9-10]. In addition to these, resolution improvement is the main challenge and key issue in MIMO imaging radar. In the point of waveform diversity view in MIMO imaging radar, using of appropriate excited pulseshapes based on dynamic variation of imaging scene makes the possibility of manipulating the incident wavefront and subsequently improving the spatial resolution [6-9].

In this paper, we explore the advantages of employing MIMO radar framework for cerebrovascular monitoring. In this context, a simulated setup of microwave head imaging system using multilayer head phantom including vascular targets and bowtie antenna is presented. The proposed bowtie antenna with precisely designed matching medium, provides a fractional bandwidth from 0.5 to $5 \mathrm{GHz}$. In order to model cerebrovascular target, a single blood vessel is simulated. The diameter of the artery is increased step-wise from $0.1 \mathrm{~mm}$ to $1 \mathrm{~mm}$. Spectrogram and range doppler plot are presented to demonstrate spectral content at different range bins which let us to detect the time-variant targets from reflected signals. Then we increase the number of targets and antennas to perform MIMO framework. In this context, we used 1D CFAR detection to demonstrate the range difference and finally in order to show different targets with different range and doppler information we applied 2D CFAR detection. Extracted results prove the effectiveness of the proposed method for precisely distinguishing time-varying targets with different dopplers and distances from antennas.

\section{Cerebrovascular MONITORING BASED ON ELECTROMAGNETIC MODALITY}

In the envisioned cerebrovascular monitoring based on electromagnetic modality frameworks such as MIMO imaging radar, it is assumed that if we could detect changes in the volume of blood vessels in different parts of the brain, we are able to demonstrate which areas of the brain are activated during performing various tasks such as visual, hearing, moving, and other activities [11]. During this neurophysiological process, oxygen and glucose consumption are increased as shown in Fig. 1 (a). Hemodynamic response yields dilation of blood vessels around $20-50 \%$ and subsequently increasing cerebral blood volume $<6 \%$ [12]. In addition, a simplified spherical model with vascular targets which is used for simulation is shown in Fig. 1 (b). 

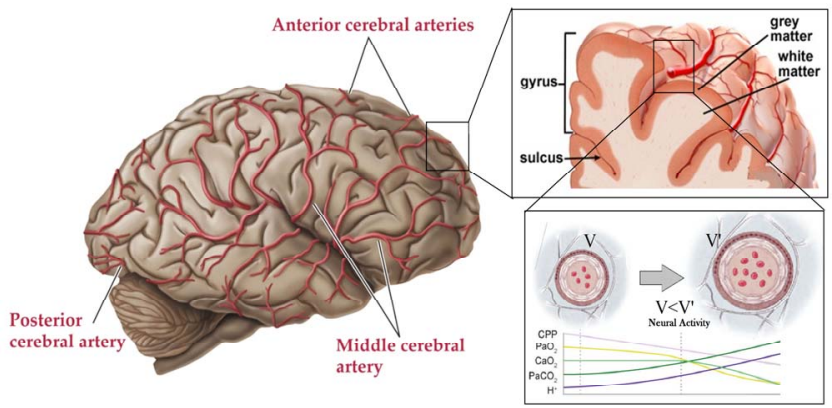

(a)

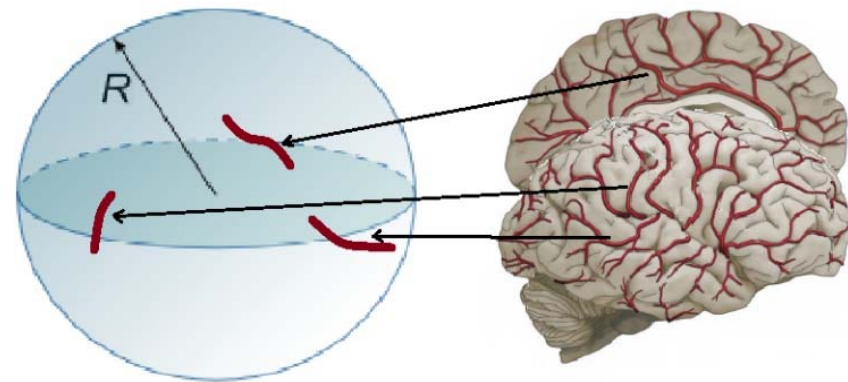

(b)

Fig. 1. (a) Brain blood vasculature as a function of blood flow [6], and (b) Converting cerbrovascular to a simlifiled spherical simulation model.

The down-range resolution which is defined as half the pulse-width is the limitation of ultra-wideband (UWB) pulse radar resolution. Down-range resolution in tissue is defined as $\Delta R=c T / 2 \sqrt{\varepsilon_{r}}$, where $T$ is the pulse duration and $\mathrm{c}$ is the light speed. Regarding to the selected frequency bandwidth for this study, required down-range resolution is possible for cerebrovascular target's motion. In order to increase the performance of system's detection in the ranging accuracy and the ability to distinguish between two targets which are really close together, the cross-range resolution should be improved. Cross range (spatial) resolution can be enhanced by increasing the antenna number and using beamforming based on waveform diversity in MIMO radar approach. It has been demonstrated that in the mm-scale targets the sub-mm variations is sensible [12]. The crossrange resolution $\Delta R_{c}$ in air is identified by: $\Delta R_{c}=\lambda / 2 \Omega$ where $\lambda$ corresponds to the wavelength of the center frequency, and $\Omega$ is the angular beamwidth (or array aperture) [9]. In addition, in MIMO framework, high frame rates for improving temporal resolution can be obtained using m-sequence pseudo noise signals for convincing temporal resolution [13].

In this study, for extracting cerebrovascular variations the range-doppler frame is generated in MIMO radar. First, fast Fourier transform (FFT) is applied for range information. Then, the second FFT is applied for doppler information extraction. Fig. 2 shows the procedure for generating range doppler profile which includes of the two-step signal processing [13]. A sequence of time domain received signals is located in each raw of the data matrix. The range profile can be obtained by applying FFT along the "fast-time". In order to extract the range-doppler profiles, windowing is applied to the range profile and after, the FFT is performed along the "slow-time". Range-doppler frames reveals moving properties, as well as micro-doppler properties of targets [13].

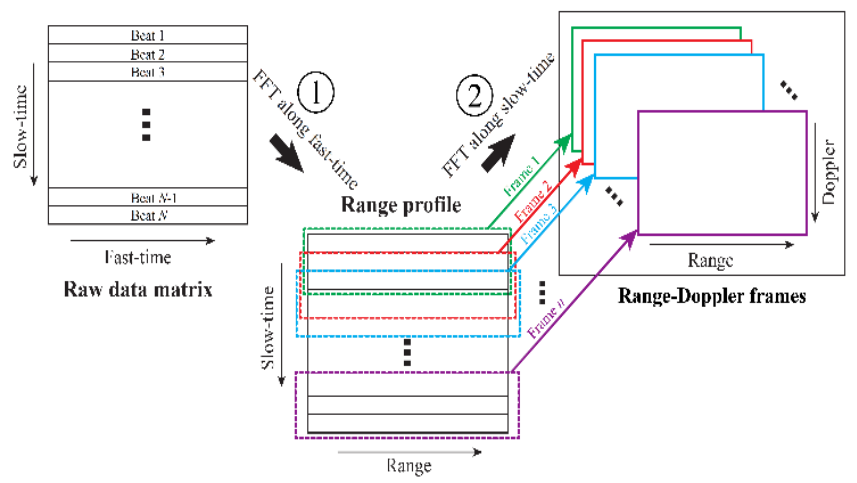

Fig. 2. Range-doppler frame generation from raw data of simulated brain activities scenario [13].

\section{CONFIGURATION OF THE PROPOSED SYSTEM}

In this section, the precisely multi-layer model of the human head phantom including cerebrovascular time-variant targets as shown in Fig. 3, is simulated in CST microwave studio [14]. The external radius of the elliptical head phantom is 12 $\mathrm{cm}$ and a cylindrical curved target with variable diameter which is located in the cerebrospinal fluid (CSF) layer of the head model is considered as cerebrovascular target. The utilized head phantom contains all anatomical details of the human head including head layers, from skin layer to white matter of the brain for ease of modeling and imaging. All electrical characteristics of the utilized head phantom's materials are given in Table I. In addition, as shown in Fig. 3 (b), the proposed antenna located in the front of the full head model at equal distances $10 \mathrm{~mm}$ from skin layer, and a blood vessel that located inside the head.

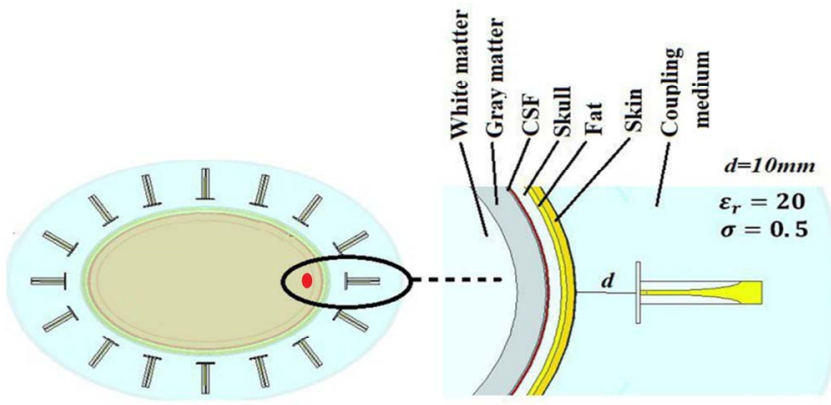

(a)

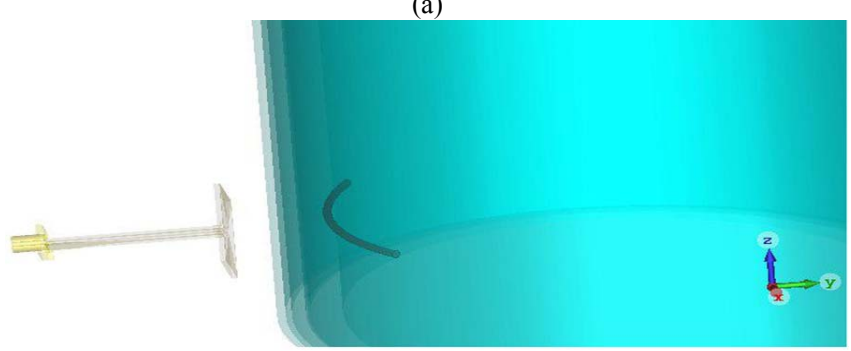

(b)

Fig. 3. (a) Multi-static UWB microwave imaging system schematic including multi layer structure of the designed head phantom and antenna's positions.for cerbrovacular target detection (front view), and (b) simulated head model and atenna with a single blood vessel in CST medium (prespective view). 
TABLE I. ELECTRICAL CHARACTERITICS OF THE MULTI-LAYER BRAIN PHANTOM

\begin{tabular}{|l|c|c|c|}
\hline \multicolumn{1}{|c|}{ Tissue } & $\begin{array}{c}\text { Thickness } \\
\text { (mm) }\end{array}$ & $\begin{array}{c}\text { Relative } \\
\text { Permittivity }\end{array}$ & $\begin{array}{c}\text { Conductivity } \\
\text { (S/m) }\end{array}$ \\
\hline Skin (dry) & 2 & 40.93 & 0.89 \\
\hline Fat & 1.4 & 5.44 & 0.05 \\
\hline Skull & 4.1 & 12.36 & 0.15 \\
\hline $\begin{array}{l}\text { Cerebrospinal } \\
\text { fluid (CSF) }\end{array}$ & 0.5 & 68.43 & 2.45 \\
\hline Gray Matter & 7 & 52.28 & 0.98 \\
\hline White Matter & Inner Part & 38.57 & 0.62 \\
\hline
\end{tabular}

For the radiating ellemnt, here we use the matched bowtie antenna which present desirable radiation and physical features, such as omnidirectioanl radiation pattern, relativly high gain, low profile strcture [15]. The proposed structure is depicted in Fig 4 which is designed based on the antennas presented previously in Ref. [15-16], but improved radiation characterstics and lower dimensions. The bow-tie antenna fed by a $50 \Omega$ microstrip line, which is printed on an Rogress 5880 substrate with the dimension of $22 \times 22 \mathrm{~mm}^{2}$, the electrical permitivity of 2.2, and the loss tangent of 0.001 . The basic antenna structure consists of a radiating patch, a balun type feed line. All dimensions of the antenna are given in Table II.

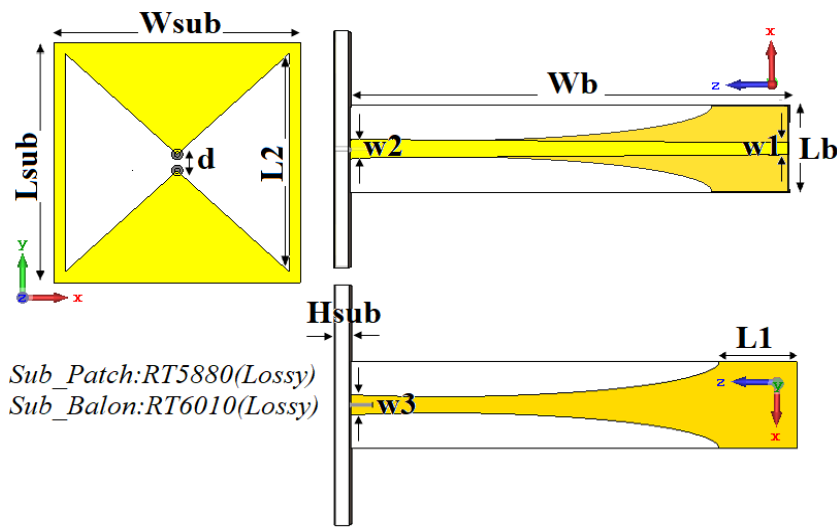

Fig. 4. The proposed matched bowtie antenna with matching balun schematic [13].

TABLE II. THE PROPOSED SLOT ANTENNA DIMENSIONS

\begin{tabular}{|c|c|c|c|}
\hline Parameter & $(\mathbf{m m})$ & Parameter & (mm) \\
\hline $\mathrm{Wsub}$ & 22 & Lsub & 22 \\
\hline $\mathrm{Wb}$ & 40 & $\mathrm{Lb}$ & 8 \\
\hline $\mathrm{W} 1$ & 1.1 & $\mathrm{~W} 2$ & 1.75 \\
\hline $\mathrm{W} 3$ & 1.84 & $\mathrm{~L} 1$ & 7 \\
\hline $\mathrm{L} 2$ & 20 & $\mathrm{Hsub}$ & 1.5 \\
\hline $\mathrm{H}_{\text {sub }}$ (Balloon) & 1.27 & $\mathrm{~d}$ & 1.5 \\
\hline
\end{tabular}

The first step before starting simulation of the microwave imaging setup is designing a matching medium. By shielding antenna in the matching medium, it is possible to decrease the mismatch effects between antenna and head phantom [15]. To ensure electrical matching between antennas and internal of the region under test a coupling medium is designed based on parametric sweep analysis of its electrical characteristics. Fig. 5 shows the comparison of the simulated return loss characteristics of the proposed antenna in different scenarios. It can be seen from Fig. 5 that by determining a good choice for permittivity and conductivity of the matching medium the antenna will be radiated from 0.5 to $5 \mathrm{GHz}$. The calculated electrical characteristics of the coupling medium are $\varepsilon_{r}=20$ and $\sigma=0.5 \mathrm{~S} / \mathrm{m}$. The frequency ranges of 0.5 to $5 \mathrm{GHz}$, shall offer a balance between the two conflicting requirements of better spatial resolution and higher penetration depths in cerebrovascular monitoring applications. By this condition the reachable theoretical spatial resolution is determined around $2 \mathrm{~mm}$, which is better than the functional magnetic resonance imaging (fMRI) systems' spatial resolution $(3-5 \mathrm{~mm})$.

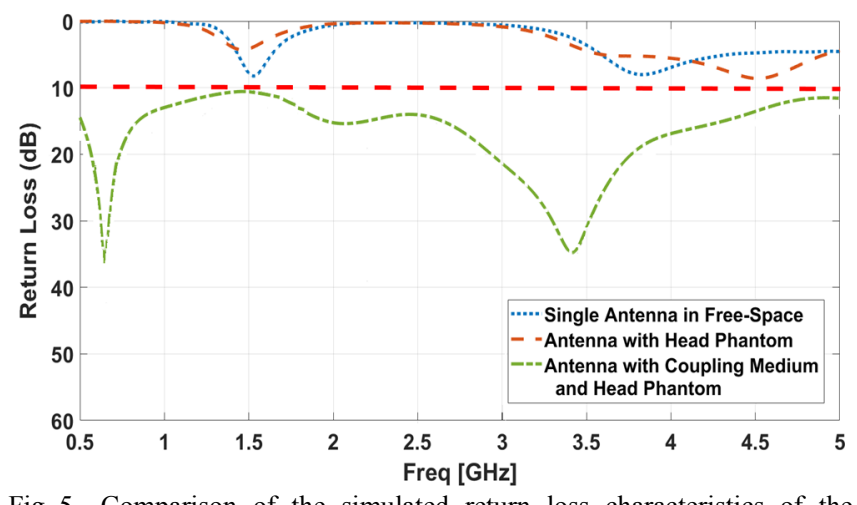

Fig. 5. Comparison of the simulated return loss characteristics of the proposed antenna in different scenarios.

\section{RESULTS AND VALIDATION DISCUSSIONS}

\section{A. Detection of Signle Blood Vessel Dilation using} Spectrogram Processing

In order to evaluate the performance of the proposed dynamic imaging system, the propsed microwave imagig system simulated in CST simulator as demonstrated in Fig. 3 and a CAD tool is programmed in MATLAB to extract the doppler infromaton from recorded signals. For this purpose, the diameter of the blood vessel's diameter is increased from 0.1 $\mathrm{mm}$ to $1 \mathrm{~mm}$ in ten steps, to gather the received signals across a wide range of blood vessel's diameters. In data processing section, first the received signals are collected by the CST studio software in frequency domain. In data acquisition process to explore the required information, several steps are applied to the received such as slow-time domain filtering, clutter removal, and inverse fast Fourier transform (IFFT) along fast time domain.

After acquisitioning of the time domain signals, this data is transferred to baseband data (I-Q). This transformation helps to extract amplitude and phase data by multiplying with the carrier frequency at a $0^{\circ}$ and $90^{\circ}$ phase shift, and arctangent demodulating. We used the ten pulse steps of the received signal and at each pulse, the blood vessel diameter is increased. By considering that the radar operates at 0.5-5 $\mathrm{GHz}$ with a gaussian pulse, Fig. 6 (a) shows the spectrogram (time-frequency response). As illustrated in Fig. 6 (a), a moving target introduces a frequency shift in the radar return due to doppler effect. In addition, Fig. 6 (b) shows the extracted range doppler plot which is highlighted the doppler changing during 0.8 seconds of scanning period. 


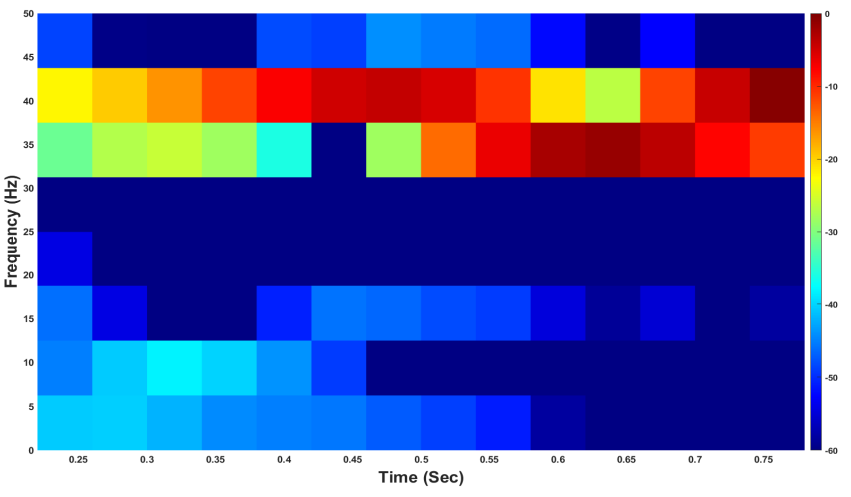

(a)

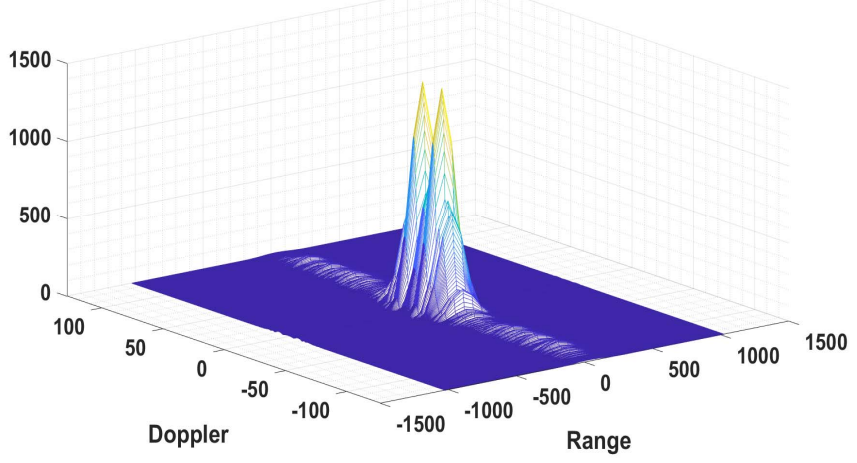

(b)

Fig. 6. Detection od signle blood vessel dilation (a) Spectrogram and (b) Range-doppler plot.

\section{B. Detection of Mutiple-Target using MIMO Radar}

B.1 Range-Doppler Processing for Two Targets with Different Ranges and Same Dilation Rates

In this section in order to consider multiple target scenario, we used the proposed multi-layer head model in the CST medium with two cerebrovascular targets as shown in Fig. 4. In order to realized MIMO array, one-dimensional hemispherical antenna array configuration is considered around nominal targets. The challenge here is detecting varying diameter of each blood vessel. For this, we have generated data cube from integrated reflected signals. The carrier frequency is $3 \mathrm{GHz}$ and the swept frequency is 12 GHz. Fig. 8 shows the $2 \mathrm{D} \mathrm{fft}$ surface plot of two cerberovascular targets with different distance from antenna. In order to extract range doppler profile of two cerberovascular targets with different distance from antenna, we applied one dimensional constant false alarm rate (CFAR) and the result is shown in Fig. 8. CFAR applys a dynamic thresholding process, which the detection threshold varies as a function of the curve under test. The CFAR technique estimates the level of "cell under test". Then this estimation is used to decide if the target is in the cell under test (CUT). This process loops across all the range cells and decides the presence of target based on the noise estimate [17].

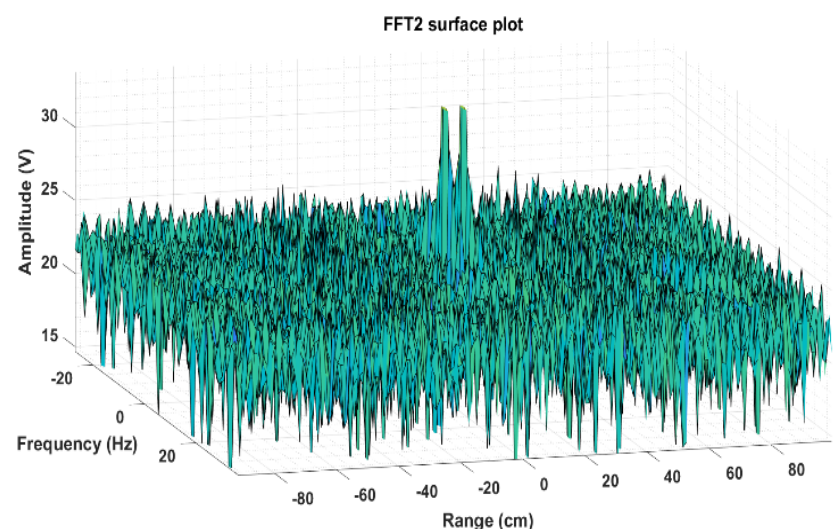

Fig. 7. 2D-FFT surface plot of two cerberovascular targets with different distance from antenna.

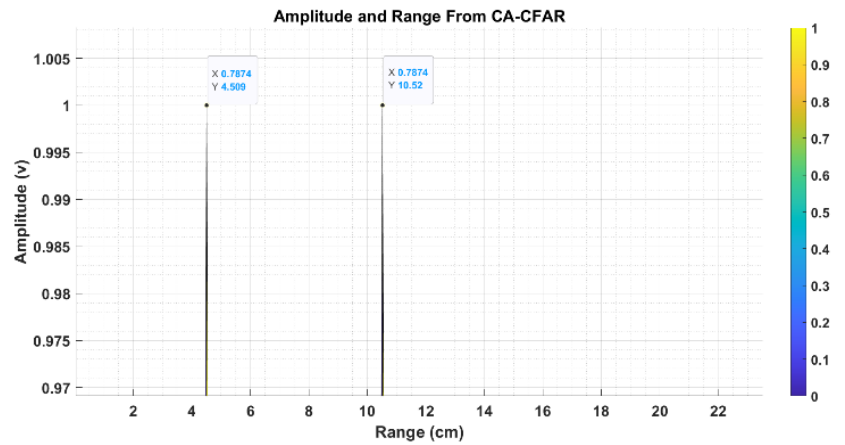

Fig. 8. Range doppler profile of two cerberovascular targets with different distance from antenna.

\section{B.2 Range-Doppler Processing for Multiple-Target Monitoring with Different Dialation Rates}

In this section a 2D-CFAR techniques are employed to detect multiple targets from range doppler extracted from data cube. Fig. 9 shows 3D range-doppler profile of two targets with different dilation rates. In addition, Fig. 10 shows range doppler profile before and after 2D CFAR detection. From these results, we found that using MIMO can get a relative higher resolution.

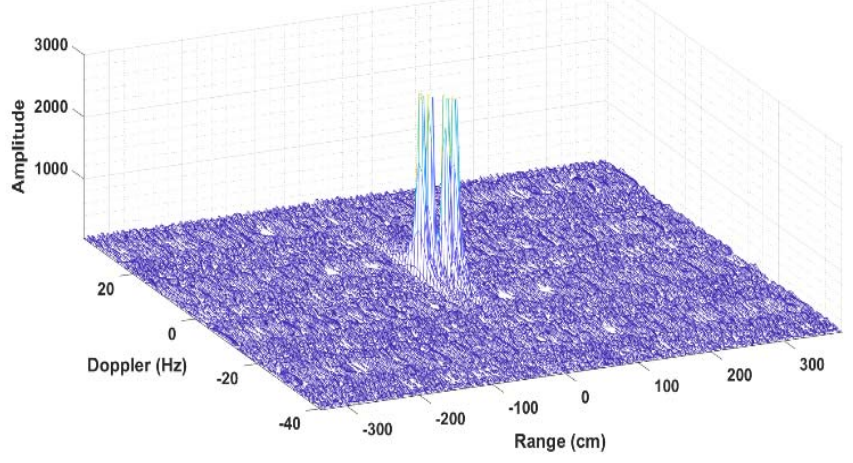

Fig. 9. The 3D Range doppler profile of two targets with different dilation rates. 


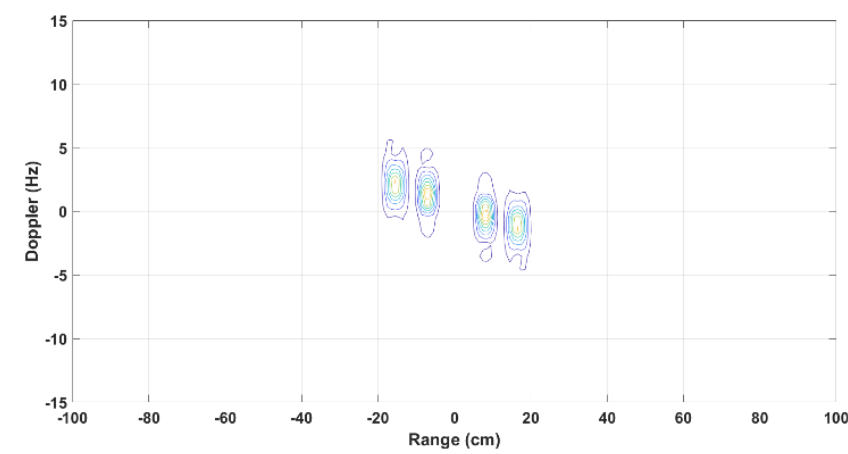

(a)

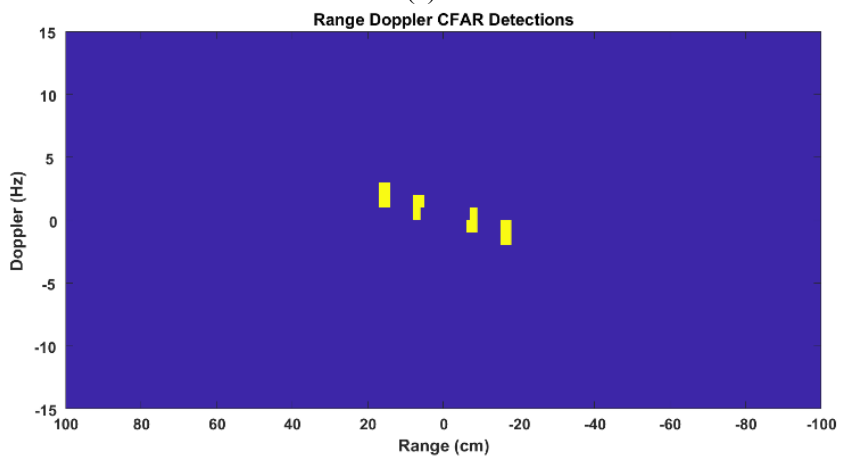

(b)

Fig. 10. Range doppler profile (a) before and (b) after 2D-CFAR detection.

\section{CONCLUSION}

This paper presents simulation setup of the MIMO microwave head imaging system using multilayer brain phantom and bow-tie antenna for physiological information extraction from cerebrovascular activities. For this purpose, reflected signals have been collected through an antenna from three different simulation scenarios which are used to extract cerebrovascular features. Spectrogram and range-doppler plot are presented to demonstrate spectral content at different range bins which let us to detect this time varying target from reflected signals. Then we increased the number of targets and antennas to perform MIMO framework. In this context, we used 1D-CFAR detection to demonstrate the range difference. Finally, in order to show different targets with different range and doppler information we applied 2DCFAR detection. Simulated results validate the effectiveness of the proposed method for precisely distinguishing timevarying targets with different doppler and distance from antennas. The results show that the proposed algorithm is able to detect cerebrovascular information in different scenarios.

\section{REFERENCES}

[1] T. Ph. Nguyen, and T. T. Tran. "Microwave Doppler Radar Sensing System for Vital Sign Detection: From Evaluated Accuracy Models to the Intelligent System." In Proceedings of the 2020 Intelligent on Intelligent Cross-Data Analysis and Retrieval Workshop, pp. 3-8. 2020.

[2] P. P. Malla, S. Sahu, and S. Routray. "Investigation of Breast Tumor Detection Using Microwave Imaging Technique." In 2020 International Conference on Computer Communication and Informatics (ICCCI), pp. 1-4, 2020.
[3] M. Ojaroudi, S. Bila, and M. Salimi, "A Novel Approach of Brain Tumour Detection Using Miniaturized High-Fidelity UWB Slot Antenna Array", (Accepted) 13th European Conference on Antennas and Propagation (EUCAP),31 March-5 April, Krakow, Poland, 2019.

[4] W. Shao, and T. McCollough. "Advances in Microwave Near-Field Imaging: Prototypes, Systems, and Applications." IEEE Microwave Magazine 21, no. 5, pp. 94-119, 2020.

[5] V.L. Coli, P. H. Tournier, V. Dolean, I. El Kanfoud, C. Pichot, C. Migliaccio, and L. Blanc-Féraud, "Detection of simulated brain strokes using microwave tomography." IEEE Journal of Electromagnetics, $R F$ and Microwaves in Medicine and Biology, 3(4), pp.254-260, 2019.

[6] M. Ojaroudi, S. Bila, Ph. Leveque, and Ph. Carré. "Functional Microwave Imagining System based on Cognitive Scanning for Brain Activities Monitoring: A Feasibility Study." In 2019 13th European Conference on Antennas and Propagation (EuCAP), pp. 1-5, 2019.

[7] J. Sachs, S. Ley, T. Just, S. Chamaani, and M. Helbig. "Differential ultra-wideband microwave imaging: Principle application challenges." Sensors 18, no. 7, 2136, 2018.

[8] M. Ojaroudi, and S. Bila, "Dynamic Short-Range Sensing Approach Using MIMO Radar for Brain Activities Monitoring", 14th European Conference on Antennas and Propagation (EUCAP), Copenhagen, Denmark, 2020. Copenhagen, Denmark15-20 March, 2020.

[9] Y. Qi, W. Yanping, W. Tan, and W. Hong. "Application of sparse array and MIMO in near-range microwave imaging." In SAR Image Analysis, Modeling, and Techniques XI, vol. 8179, p. 81790X International Society for Optics and Photonics, 2011.

[10] O. Fiser, M. Helbig, J. Sachs, S. Ley, I. Merunka, and J. Vrba, "Microwave non-invasive temperature monitoring using UWB radar for cancer treatment by hyperthermia". Progress In Electromagnetics Research, 162, pp.1-14, 2018.

[11] T. Lauteslager, N. Nicolaou, T. S. Lande and T. Constandinou, Functional neuroimaging using UWB impulse radar: A feasibility study. In Biomedical Circuits and Systems Conference (BioCAS), 2015 IEEE (pp. 1-4), October 2015.

[12] T. Lauteslager, M. Tømmer, T. S. Lande, and T. Constandinou, "Coherent UWB Radar-on-Chip for In-Body Measurement of Cardiovascular Dynamics." IEEE transactions on biomedical circuits and systems, 2019.

[13] T. Miwa, S. Ogiwara, and Y. Yamakoshi, MIMO radar system for respiratory monitoring using $\mathrm{Tx}$ and $\mathrm{Rx}$ modulation with M-seauence codes. IEICE transactions on communications, 93(9), pp.2416-2423, 2010.

[14] CST Microwave Studio. ver. 2019, CST, Framingham, MA, USA, 2019.

[15] M. Ojaroudi, S. Bila, and M. Salimi, "A Novel Machine Learning Approach of Haemorrhage Stroke Detection in Differential Microwave Head Imaging System", Accepted for publication in 14th European Conference on Antennas and Propagation (EUCAP), Copenhagen, Denmark, 15-20 March, 2020. Copenhagen, Denmark, 2020.

[16] M. Jalilvand, C. Vasanelli, Ch. Wu, J. Kowalewski, and Th. Zwick. "On the evaluation of a proposed bowtie antenna for microwave tomography." In The 8th European Conference on Antennas and Propagation (EuCAP 2014), pp. 2790-2794, 2014.

[17] S. K. Joshi, and S. V. Baumgartner. "Training Data Selection Strategy for CFAR Ship Detection in Range-Compressed Radar Data." In 2019 International Radar Conference (RADAR), pp. 1-5, 2019. 